\title{
Measurement of BacT/ALERT Sensitivity after Inoculation Certain Amount of E. coli and S. epidermis
}

\author{
Abolfazl Dabirmoghadam ${ }^{1}$, Farhad Razjou ${ }^{1^{*}}$ and Bahram A. Badlou ${ }^{2^{*}}$ \\ ${ }^{1}$ Blood Transfusion Research Center, High Institute for Research and Education in Transfusion Medicine, IBTO, Tehran, Iran \\ ${ }^{2}$ BBAdvies and Research, Research and Development Dep. Zeist, The Netherlands
}

*Corresponding authors: Razjou F, PhD Pathology, Blood Transfusion Research Center, High Institute for Research and Education in Transfusion Medicine, IBTO, Tehran, Iran, Tel: +982188602180; E-mail: frazjou@yahoo.com

Bahram A. Badlou (PhD), BBAdvies and Research, Research and Development Dep. Zeist, The Netherlands, Tel: +31302211328; E-mail: bbadlou@casema.nl

Received date: Mar 02, 2015, Accepted date: Apr 11, 2015, Publication date: Apr 15, 2015

Copyright: (c) 2015 Dabirmoghadam A, et al. This is an open-access article distributed under the terms of the Creative Commons Attribution License, which permits unrestricted use, distribution, and reproduction in any medium, provided the original author and source are credited.

\section{Abstract}

Background: Transfusion of contaminated human platelets concentrates (PCs) causes septic reaction and death of recipients. Current rate of detection of bacterial-contaminated of the PCs are very important tools to warranty patients'safety. BacT/ALERT system was introduced as one of the most sensitive, specific, and rapid screening test, which could be implemented in Blood Transfusion Centers.

The aim of this study is to investigate whether the BacT/ALERT system as introduced, can function as rapid detection system

Material and Methods: In this study we used the whole blood from 24 healthy human subjects with inform consent, The PCs were collected from these whole blood samples by differential Centrifugation and Buffy coat system. All PCs (50-70 mL) stored under standard blood banking conditions. The PCs were randomly selected and divided in two groups of 12 , and $10 \mathrm{CFU} / \mathrm{mL}$ was of either $E$. coli (gram negative) added into first group ( $\mathrm{n}=12 \mathrm{PCs}$ ), or S. epidermidis (gram positive) bacteria into another group ( $n=12$ PCs). Then samples were inoculated in BPA culture medium of BacT/ALERT system and studied after $0,6,24,48 \mathrm{hrs}$.

Results: The BacT/ALERT system showed rapidly positive results with E. coli samples (T0) with all introduced volumes $(0.5,1,2 \mathrm{ml})$ but with $S$. epidermidis samples showed $83 \%$ with low volumes $(0.5$ and $1 \mathrm{ml})$, and $91.6 \%$ positive alert results with high volume $(2 \mathrm{ml})$ after $48 \mathrm{hrs}$ incubations.

Conclusion: This indicates that the sensitivity and specificity of BacT/ALERT system is disputable and not universal, which remained to be investigated in details.

Keywords: Blood transfusion; Bacterial screening; E. coli; Transfusion transmitted diseases, S. epidermidis, Microbiology

\section{Introduction}

Transfusion of contaminated human platelet concentrates (PCs) results in transfusion transmitted diseases (TTD), septic reaction and death of recipients, however [1-3]. Detection methods of bacterial contamination of PCs are very important tools to warranty patients'safety $[2,4]$. The TTD and septic reactions steadily are reported with culture-tested PCs, possibly due to false-negative outcome associated with inadequate sampling of low-concentration of certain bacteria [3]. Current detection technologies to screen bacterial contamination of blood products are rather aspecific, not $100 \%$ sensitive, and relatively lethargic. Hence, these failures should question the usefulness of systematic bacterial detection of the PCs, when the lethal accidents are occurring after routine controls $[5,6]$.

Recently developed automated techniques for detection of bacteria are much more rapid than direct plating techniques, and the blood culture $[4,7]$. One assumes that such rapid techniques can be useful to monitor the sterility of cellular blood products with greater sensitivity, using a small aliquot of the blood product taken soon after collection [8].

Venipuncture during blood collection is probably the major cause of bacterial contamination of blood components since the majority of contaminant organisms are part of the normal or transient skin flora [9]. To reduce hazardous of bacterial contamination and proliferation it is important to implement a rapid and highly specific detecting test in every Blood Bank. Globally, microbial infection of blood products is a determined but often ignored problem in the Blood Transfusion Centers [10]. The possible sources of microbial contamination of blood products consist of different origin i.e. donor bacteremia, 5 during and after blood collection procedure, from the collection pack, during the blood manufacturing process and storage temperature $\left(20-24^{\circ} \mathrm{C}\right)[11]$.

Unfortunately still the most frequent TTDs 'complication occurs with PCs transfusion with an incidence between 1:1000 and 1:3000 $[10,12]$. Obviously, dissimilarities in transfusion reactions against bacterial inocula transfused, lack of standard procedure, and at least but not last lack of $100 \%$ sensitive/specific machines affected 'rate of detection' [13]. Timely detection of contaminated PCs play pivotal role 
in the fundaments of cascade of failures, which might unpurposely expose hemato-oncologic patients to unknown dosis of microorganisms posttransfusion [14]. After recognition of lethal effects of contaminated PCs prompted the College of American Pathologists (CAP) and the American Association of Blood Banks (AABB) to set new standards requiring the screening of PCs for bacterial contamination $[13,15]$.

The aim of this study is to investigate whether BacT/ALERT system as introduced, can function as relaible detection system under our circumstances. Furthermore, to evaluate 'rate of detection' by introducing different volume of contaminated PCs innoculated with known endconcentration of certain bacteria i.e. E. coli and $S$. epidermidis.

Here we report that after introducing different PCs inoculated with known concentration of certain bacteria BacT/ALERT system showed positive results immidiately after adding $10 \mathrm{CFU} / \mathrm{mL}$ but it obviouly works sort-dependent.

\section{Materials and Study Design}

This descriptive research study was done with $1 \%$ of daily processed PCs in the IBTO from 24 healthy donors whole blood' sample with inform consent, which were separated by differential centrifugation into PCs. All processed PCs $(\mathrm{n}=24)$ containing $50-70 \mathrm{ml}$ volume stored under standard blood banking's conditions at $22-24^{\circ} \mathrm{C}$ with agitation, prior to detection tests (Figure 1).

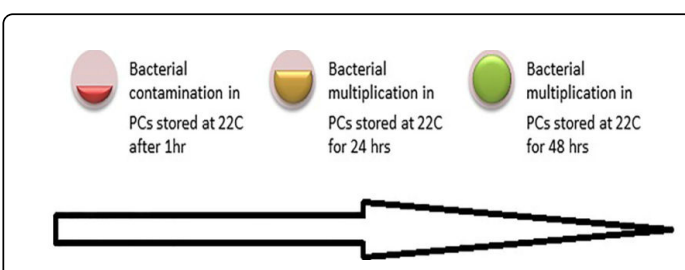

Figure 1: The human platelets concentrates (PCs) contamination and multiplication processes after 48 hours storage.

In order to improve the diagnosis of bacterial contamination, we used the BacT/ALERT system to screen bacterial infections of PCs (6). The BacT/ALERT system is alarming when minimal endconcentration of certain microorganism (inoculum) reaches $10 \mathrm{CFU} / \mathrm{mL}$ in blood products, and its alert signal is based on $\mathrm{CO}_{2}$ emissions in the culture medium (colorimetric technology/ sensor culture bottles) [16]. The system with the use of specific media that is embeded in the device, allows continuous monitoring of cultivation via the display of the BacT/ALERT system. Two relevant bacteria i.e. E. coli and $S$. epidermidis strains were isolated and purified for this study, and were confirmed by biochemical reactions as described [17]. Both contaminants were prepared for PCs specifically. Then, fresh cultures of bacteria isolated from the colonies and inoculated into the Trypticase broth environment (Construction company, Merck, Germany). Subsequently, the new colonies of the bacteria were prepared in a standard fashion i.e. $0.5 \mathrm{McF}$ arland, $10 \mathrm{CFU} / \mathrm{mL}$ aliquote dilutions (Figure 2). Bacteria populations were counted and 10 $\mathrm{CFU} / \mathrm{mL}$ was added to each randomly selected PC, which supposedly high enough to be detected by BacT/ALERT system as $\mathrm{CO}_{2}$ release causes sensor bottle to turn yellow, Instrument measures and detects color change, analyzes data to determine positivity, alerts when culture is positive.(5) Next different harvested volumes (0.5, 1, $2 \mathrm{~mL}$ ) (Figure
3A-3C) were used for each inoculation, under sterile conditions. The PCs were inoculated with the certain bacteria, which commonly contaminate PCs pretransfusion i.e. E. coli and $S$. epidermidis. Furthermore, randomly selected PCs were incubated with BPA medium under defined condition, and then daily the samples were checked by employing BacT/ALERT standard bottles BPA (BioMerieux, Inc., Durham, NC) for detection of aerobic bacteria. The BacT/ALERT's bacterial display after the first alarm automatically records all positive results.

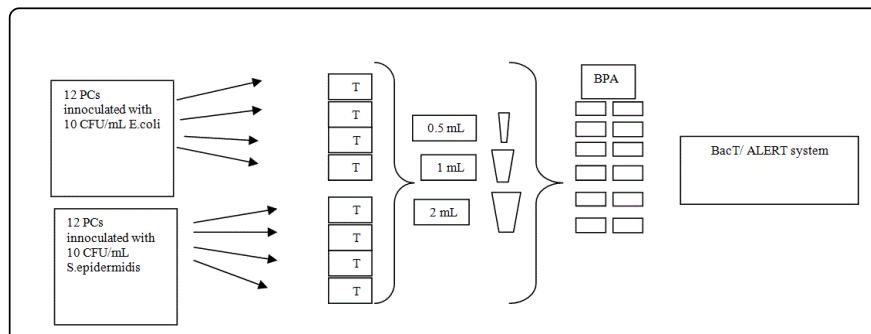

Figure 2: Bacterial contamination tests with known artificially made contaminants.
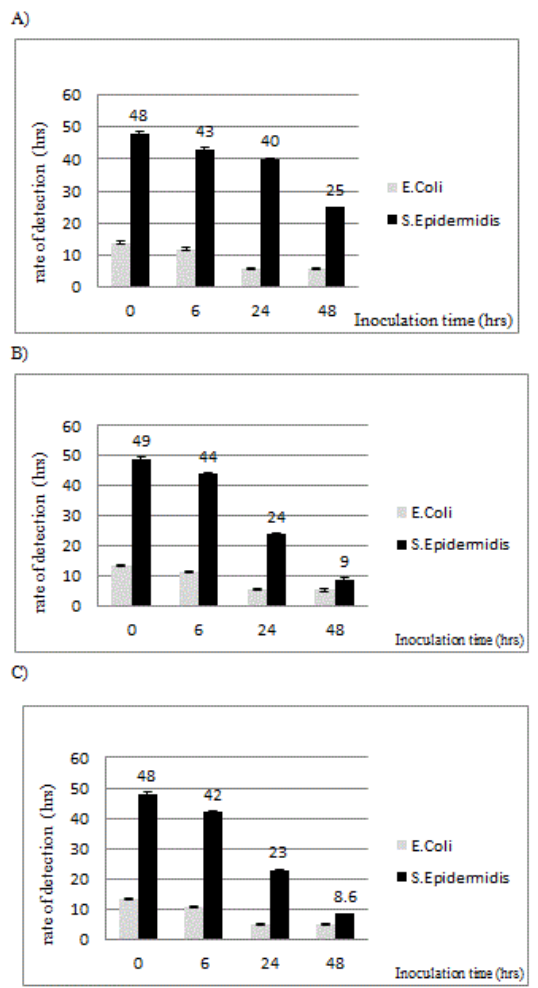

Figure 3: Rate of detection of positive results of BacT/ALERT system of contaminated PCs with A) 0,5, B) 1 , and C) $2 \mathrm{~mL}$ volume of certain known bacteria i.e. E. coli and $S$. epidermidis (10 $\mathrm{CFU} / \mathrm{mL}$ )

Acquired data were analyzed by ANOVA test and $\mathrm{p}<0.05$ was considered significant $(\mathrm{n}=288)$. 


\section{Results}

Because E. coli (fast) and S. epidermidis (slow) have different growth rates, the results of our studies gathered, and compared with each others. Due to the multifactorial aspects of detection tests i.e. rate of detection, sensitively, and specificity the capapbility of the BacT/ ALERT system was investigated in the IBTO. We set priorities and focused on detection alarm rate after addition of three different volumes $0,5,1$, and $2 \mathrm{~mL}$ (Figure $3 \mathrm{~A}-3 \mathrm{C}$ ) contaminants containing at least a begin concentration of $10 \mathrm{CFU} / \mathrm{mL}$ of $E$. coli and/or $S$. epidermidis at time point zero (T0).

Comparison of different measurements showed that the BacT/ ALERT system displayed with different rate a positive results when the PCs were inoculated with $0.5 \mathrm{~mL}$. Immidiately after incubation (T0) (Control groups 1, C1), BacT/ALERT system significantly $(\mathrm{p}<0.05)$ displayed the $E$. coli samples much quiker as positive than $S$. epidermidis (rate of detection $>10 \mathrm{hrs}$ after $10 \mathrm{CFU} / \mathrm{mL}$ ). Moreover, compared to $\mathrm{C} 1$ after $6,24,48 \mathrm{hrs}$ inoculation we observed again $\mathrm{E}$. coli was quicker detected than S. epidermidis, respectively.

In the case of $S$. epidermidis immidiately after incubation (T0) (control groups 2, C2), BacT/ALERT system displayed positive with 0.5 and $1 \mathrm{ml}$, only 10 cases, while with $2 \mathrm{~mL}$ volume 11 of 12 cases. However, The BacT/ALERT system displayed contaminated samples for $100 \%$ positive (12 of 12 ) after 6,24 , and $48 \mathrm{hrs}$ incubation with all volumes.

As it is shown in the Figure 3B the BacT/ALERT system displayed with different rates a positive results when PCs were inoculated with 1 $\mathrm{mL}$. Inoculation time from 6 up to $48 \mathrm{hrs}$ showed inverse relation versus rate of detection displayed by the BacT/ALERT system. Obviously, multiplication of bacteria increases when more adjucent bacteria is present irrespective of bacteria sort.

Compared to control group, the mean rate of detection reduced to 5 hrs when $2 \mathrm{~mL}$ of $E$. coli-contamnated PCs $48 \mathrm{hrs}$ was incolated (Figure 3C). With other words, the amount of bacteria when passes a certain limit, rate of detection time decreases, signifantly. And conrorevise, when the amount is below $10 \mathrm{CFU} / \mathrm{mL}$, tempo of detetcion alarm delayed considerably, as such that using of the BacT/ ALERT system become redundant for the Blood bank. Beside, analysis of between-groups showed that a sort-dependent detection mechanism dominated the BacT/ALERT system. Furthermore, we observed that how longer PCs' inoculation time was extended how the rate of detection was increased. Analysis of within-groups showed that how higher the volume of contaminants how earlier the BacT/ALERT system gave positive alert signal. When PCs were inoculated with highst volume $(2 \mathrm{~mL})$ the BacT/ALERT system displayed with different rates a positive results (Figure 3 ).

Nonetheless BacT/ALERT system failed to detect minimum of $S$. epidermidis $(10 \mathrm{CFU} / \mathrm{mL})$ even after addition of $2 \mathrm{~mL}$ to solutions, ealier than $48 \mathrm{hrs}$ (at T0).

\section{Discussion}

In this study random human PCs inoculated with known final concentration $(10 \mathrm{CFU} / \mathrm{mL})$ of known bacteria (E. coli and $S$. epidermidis) to investigate specificity and sensitivity of BacT/ALERT system. Compared to control group after 24 inuculation of E. coli the BacT/ALERT system displayed a $100 \%$ sensitivity but $83.3 \%$ specificity. The BacT/ALERT system failed to detect in the first $10 \mathrm{hrs}$ to display any infected sample as positive, even after adding $2 \mathrm{~mL}$ contaminants. From 144 with E. coli inoculated PCs samples the BacT/ ALERT system could measure all (100\%), while with $S$. epidermidis $98 \%$ were alerted as positive, consequently missed $2 \%(n=28$; false negative).

These data confirm other laboratory findings. Why in our study 2 samples of 12 at $\mathrm{T} 0$ were not displayed as positive might be caused by either failure of the BacT/ALERT system or something else, which needs more investigations.

Data acquired were from 24 samples shows that BacT/ALERT system has not $100 \%$ sensitivity tested by known final concentration of $S$. epidermidis $(10 \mathrm{CFU} / \mathrm{mL})$. Unforyunately this percentage is too high when patients safety and costeffectiness concern play pivotal rule to use this system.

In fact every Blood bank is expecting to have a rapid bacterial detection test that warranty any blood products'sterility and safty. In one hand the BacT/ALERT system was partially able to alert us in less than $10 \mathrm{hrs}$. In the other hand rate of detection time efficacy, and costeffectiveness of the BacT/ALERT system studied were not favorable. One may speculate what would be the reason to buy such expensive equipement with so specificity and sensitivity.

Taken together, our results at least have shown that the BacT/ ALERT system under different circumstances should be tested again. Despite the consequences on patients safety the BacT/ALERT displayed a dose- and sort dependent results at its best condition. The rate of detection was not so impressive and missed positive samples, which might not a justifiable issue. In our opion, based on this scores of the BacT/ALERT system, the Blood Banks and Transfusion Centers take high responsiblities for patients'safety.

\section{References}

1. Eder AF, Goldman M (2011) How do I investigate septic transfusion reactions and blood donors with culture-positive platelet donations? below Transfusion 51: 1662-1668.

2. Blajchman MA (1995) Bacterial contamination of blood products and the value of pre-transfusion testing. below Immunol Invest 24: 163-170.

3. Benjamin RJ, Wagner SJ (2007) The residual risk of sepsis: modeling the effect of concentration on bacterial detection in two-bottle culture systems and an estimation of false-negative culture rates. Transfusion 47: 1381-1389.

4. Mastronardi C, Perkins H, Derksen P, denAdmirant M, Ramírez-Arcos S (2010) Evaluation of the BacT/ALERT 3D system for the implementation of in-house quality control sterility testing at Canadian Blood Services. below Clin Chem Lab Med 48: 1179-1187.

5. Courbil R, Romaszko JP, Odent-Malaure H, Fabrigli P, Chavarin P, et al. (2010) [Analysis of a severe septic transfusion reaction with standard platelet concentrate]. below Transfus Clin Biol 17: 9-13.

6. Hillyer CD, Josephson CD, Blajchman MA, Vostal JG, Epstein JS, et al. (2003) Bacterial contamination of blood components: risks, strategies, and regulation: joint $\mathrm{ASH}$ and $\mathrm{AABB}$ educational session in transfusion medicine. Hematology Am Soc Hematol Educ Program 2003: 575-89.

7. Benjamin RJ, Kline L, Dy BA, Kennedy J, Pisciotto P, et al. (2008) Bacterial contamination of whole-blood-derived platelets: the introduction of sample diversion and prestorage pooling with culture testing in the American Red Cross. Transfusion 48: 2348-2355.

8. Blajchman MA, Goldman M (2001) Bacterial contamination of platelet concentrates: incidence, significance, and prevention. below Semin Hematol 38: 20-26.

9. Ramírez-Arcos S, Jenkins C, Dion J, Bernier F, Delage G, et al. (2007) Canadian experience with detection of bacterial contamination in apheresis platelets. Transfusion 47: 421-429. 
Citation: Dabirmoghadam A, Razjou F, Badlou BA (2015) Measurement of BacT/ALERT Sensitivity after Inoculation Certain Amount of E. coli and S. epidermis. J Blood Disorders Transf 6: 268. doi:10.4172/2155-9864.1000268

Page 4 of 4

10. Brecher ME, Blajchman MA, Yomtovian R, Ness P, AuBuchon JP (2013) Addressing the risk of bacterial contamination of platelets within the United States: a history to help illuminate the future. below Transfusion 53: 221-231.

11. Blajchman MA, Goldman M, Baeza F (2004) Improving the bacteriological safety of platelet transfusions. Transfus Med Rev 18: 11-24.

12. Yomtovian R, Jacobs MR (2010) A prospective bonus of platelet storage additive solutions: a reduction in biofilm formation and improved bacterial detection during platelet storage. Transfusion 50: 2295-2300.

13. Palavecino EL, Yomtovian RA, Jacobs MR (2006) Detecting bacterial contamination in platelet products. below Clin Lab 52: 443-456.

14. Walther-Wenke G, Schrezenmeier H, Deitenbeck R, Geis G, Burkhart J, et al. (2010) Screening of platelet concentrates for bacterial contamination: spectrum of bacteria detected, proportion of transfused units, and clinical follow-up. Ann Hematol 89: 83-91.

15. Yomtovian RA, Palavecino EL, Dysktra AH, Downes KA, Morrissey AM, et al. (2006) Evolution of surveillance methods for detection of bacterial contamination of platelets in a university hospital, 1991 through 2004. below Transfusion 46: 719-730.

16. McDonald CP, Pearce S, Wilkins K, Colvin J, Robbins S, et al. (2005) Pall eBDS: an enhanced bacterial detection system for screening platelet concentrates. below Transfus Med 15: 259-268.

17. Wagner SJ, Robinette D (1998) Evaluation of an automated microbiologic blood culture device for detection of bacteria in platelet components. below Transfusion 38: 674-679. 